06

\title{
Влияние свойств границы раздела линейной и нелинейной оптических сред на потоки энергии нелинейных поверхностных волн
}

\author{
(C) C.E. Савотченко \\ Белгородский государственный технологический университет им. В.Г. Шухова, \\ 308012 Белгород, Россия \\ e-mail: savotchenkose@mail.ru
}

Поступила в редакцию 27.12.2018 г.

В окончательной редакции 27.12.2018 г.

Принята к публикации 23.01.2019 г.

\begin{abstract}
Рассмотрены процессы локализации возбуждений поля в виде нелинейных поверхностных волн, распространяющихся вдоль границы раздела линейной и нелинейной сред. Проанализированы условия существования нелинейных поверхностных волн, определяемые соотношением между линейными характеристиками сред, коэффициентом керровской нелинейности и интенсивностью взаимодействия волны с границей раздела. Рассчитаны и проанализированы зависимости потока энергии, переносимой нелинейными поверхностными волнами. Предложены два подхода к выбору управляющих параметров при определении потока энергии, в качестве которых выступают положение максимума возмущения поля в нелинейном полупространстве и значение амплитуды поля на границе раздела сред. Найдены оценки потоков в различных предельных случаях, соответствующих малоамплитудным возмущениям поля, слабому или сильному взаимодействию нелинейной поверхностной волны с границей раздела сред. Показано, что для малоамплитудных возмущений поля в случае слабого взаимодействия волны с границей поток в линейном полупространстве прямо пропорционален интенсивности взаимодействия волны с границей, а в нелинейном полупространстве обратно пропорционален ей.
\end{abstract}

DOI: $10.21883 /$ OS.2019.05.47652.380-18

\section{1. Введение}

Использование уникальных свойств поверхностных волн, том числе и нелинейных, в различных технических системах, основанных на волноводных свойствах, обусловливает неутихающий интерес к их изучению [1-4].

Особенности взаимодействия возбуждений электромагнитного поля с границами раздела сред, проявляющиеся при учете их внутренних свойств, являются существенными в тех случаях, когда они могут быть выбраны в качестве управляющих параметров, контролирующих локализацию и волноводные свойства [5]. Оптические многослойные системы с управляющими параметрами находят широкое применение в различных устройствах, в которых контролирующие различные процессы параметры позволяют устанавливать оптимальные значения пропускных характеристик границ раздела сред на определенных частотах $[6,7]$.

Как правило, управление потоками энергии, уносимыми поверхностными волнами, осуществляется с помощью модуляции показателя преломления оптических сред в слоистых структурах в поперечном слоям направлении [8]. Существует множество теоретических работ, посвященных данному направлению, в которых рассматривались нелинейные поверхностные волны оптического диапазона, распространяющиеся вдоль границ раздела сред и локализованные в поперечном им направлении [9-11].

Следует отметить, что характер нелинейности сред рассматривался не только керровский, когда диэлектри- ческая функция (или показатель преломления) зависит от интенсивности потока энергии волны, фактически определяемой квадратом амплитуды напряженности электрического поля, но и среды с иными формами зависимости диэлектрической функции [12-15].

Во многих ситуациях возникает необходимость изучения таких особенностей локализации волн вблизи границ раздела линейной и нелинейной сред, которые обусловлены характером их взаимодействия с дефектами [16-18], в том числе и для двухуровневых систем [19-21].

Здесь возникает другой подход к управлению потоками энергии, уносимыми поверхностными волнами, который может осуществляться теперь не за счет разницы между показателями преломления контактирующих сред, а с помощью конечной интенсивности взаимодействия волны непосредственно с самой границей раздела сред [22,23]. Наличие такого управляющего параметра позволяет контролировать распределение светового потока, локализация которого возможна даже в случае одинаковых значений показателей преломления контактирующих сред.

Локализации поля вблизи контракта двух нелинейных сред с одинаковыми показателями преломления, к примеру, была описана в [24]. Кроме того, увеличение эффективности управления потоками энергии поверхностных волн может достигаться в ультратонких разграничивающих среды слоях, которые характеризуются дополнительным нелинейным откликом [15,25-30]. 
Теоретическое описание распределения напряженности электрического поля в плоско поляризованной поверхностной волне приводит к нелинейному уравнению Шредингера (НУШ), которое для керровской среды содержит кубическое относительно искомого поля слагаемое [31]. Следует отметить, что НУШ широко применяется для описания не только нелинейных поверхностных волн оптического диапазона, но и полей другой физической природы, к примеру, упругого [32], магнитного [33], а также в биологических молекулярных системах [34].

В данной работе описываются особенности локализации нелинейных поверхностных волн вблизи границы раздела линейной и нелинейной оптических сред на основе НУШ. Будут описаны нелинейные поверхностные волны, возникающие в двух случаях: вблизи границы раздела линейной и нелинейной самофокусирующей сред и вблизи границы раздела линейной и нелинейной дефокусирующей сред. В явном аналитическом виде будут получены зависимости декрементов затухания волн, констант распространения, амплитуд и потоков энергии волн от таких физических характеристик сред, как их линейные показатели преломления, коэффициенты керровской нелинейности, интенсивности взаимодействия волны с границей раздела.

В данной работе большое внимание уделяется анализу законов дисперсии нелинейных поверхностных волн и уносимых ими потоков энергии с двух позиций, различающихся выбором управляющей физической характеристики. Использование таких двух подходов, в первую очередь, отличает данную работу от существующих теоретических работ [8-10], в которых также вычислялись потоки энергии волн, распространяющихся вдоль границ раздела линейных и нелинейных сред. Кроме того, в данной работе последовательно проведен подробный теоретический анализ влияния двух основных факторов, обусловливающих локализацию возмущения электрического поля вблизи границы раздела сред, таких, как различие линейных показателей преломления сред и интенсивность взаимодействия волны с границей раздела сред.

Следует подчеркнуть, что анализ влияния такой характеристики как интенсивность взаимодействия волны с границей раздела линейной и нелинейной сред ранее детально не проводился. Поэтому в данной работе учет такой характеристики является принципиальным, что и обусловливает необходимость проведения анализа влияния на поток энергии, уносимой поверхностной волной, интенсивности ее взаимодействия с границей раздела, вдоль которой она распространяется, а локализуется в перпендикулярном к ней направлении.

\section{2. Уравнения модели}

Рассмотрим контакт линейной и нелинейной керровской оптических сред, разделенных ультратонким оптическим слоем, толщина которого много меньше характерного масштаба локализации возмущений параметров среды, создаваемых им. В пределе бесконечно малой толщины такого слоя его можно рассматривать как плоскую границу раздела сред. Выберем систему координат так, чтобы данная граница раздела лежала в плоскости $y z$ и проходила через начало координат перпендикулярно оси $x$. В рассматриваемом контексте граница раздела сред играет роль волновода.

В [15] было показано, что $y$-ю компоненту напряженности электрического поля ТЕ-поляризованной монохроматической электромагнитной волны, распространяющейся вдоль границы раздела (плоскости $y z$ ), можно представить в виде $E(x, z) E(y)$, а распределение поля $E(x, z)$ будет подчиняться НУШ:

$$
i \frac{\partial E}{\partial z}+D \frac{\partial^{2} E}{\partial x^{2}}+n_{L}(x) E+n_{N}\left(x,|E|^{2}\right) E=0,
$$

где $D$ - коэффициент дифракции (далее будем считать $D=1$ для упрощения вычислений), $n_{L}(x)$ - линейный показатель преломления, который в нелинейных средах считается неизменным вдоль границы раздела и меняющимся в направлении, перпендикулярном ее плоскости (вдоль оси $x$ ), $n_{N}\left(x,|E|^{2}\right)=\theta(x) \gamma|E|^{2}-$ нелинейная добавка к нему, $\gamma$ - коэффициент керровской нелинейности среды (постоянная величина), расположенной справа от границы, $\theta(x)$ - тета-функция Хевисайда.

Стационарные состояния описываются полем $E(x, z)=u(x) \exp (-i \omega z), \quad$ где $\omega-$ константа распространения и, как следует из (1), функция $u(x)$ подчиняется стационарному НУШ, которое представим в традиционной форме [16,35]:

$$
u^{\prime \prime} / 2+\left\{\omega-\Omega(x)+\theta(x) \gamma|u|^{2}\right\} u=U(x) u,
$$

где $\Omega(x)$ определяет зависимость от координаты $x$ линейного показателя преломления сред, которую будем аппроксимировать кусочно-постоянной функцией:

$$
\Omega(x)=\left\{\begin{array}{ll}
\Omega_{L}, & x<0 \\
\Omega_{N}, & x>0
\end{array},\right.
$$

$\Omega_{L, N}$ - постоянные величины.

Функция $U(x)$ в (2) определяется параметрами взаимодействия поля с границей раздела слоев и представляет собой потенциал, моделирующий взаимодействие поля с границами раздела слоев [15]. Будем считать, что среда внутри самой границы раздела характеризуется своим линейным показателем преломления, который и определяют форму потенциала $U(x)$. Тогда в силу того, что толщина границы существенно меньше характерной длины локализации возмущений поля, локальный потенциал в короткодействующем („точечном“) приближении можно аппроксимировать дельта-функцией Дирака:

$$
U(x)=U_{0} \delta(x),
$$

где $\delta(x)$ - дельта-функция Дирака, $U_{0}-$ константа, пропорциональная линейному показателю преломления 
в ультратонкой границе раздела слоев. Величину $U_{0}$ будем интерпретировать как интенсивность взаимодействия возбуждений поля с границей раздела в линейном приближении (называемая часто „мощностью“ дефекта): при $U_{0}>0$ возбуждения отталкиваются от границы, а при $U_{0}<0$ - притягиваются.

В данной работе будет анализироваться сохраняющийся вдоль волновода поток энергии, переносимый поверхностной волной, который представляет собой первый интеграл НУШ (2):

$$
P=\int_{-\infty}^{+\infty}|u(x)|^{2} d x .
$$

Решение НУШ (2) с потенциалом (3) сводится к решению двух стационарных УШ без потенциала на полуосях:

$$
\begin{gathered}
u^{\prime \prime}(x)+2\left(\omega-\Omega_{L}\right) u(x)=0, \quad x<0, \\
u^{\prime \prime}(x)+2\left(\omega-\Omega_{N}\right) u(x)+2 \gamma|u(x)|^{2} u(x)=0, x>0,
\end{gathered}
$$

с граничными условиями:

$$
\begin{gathered}
u(-0)=u(+0)=u_{0}, \\
u^{\prime}(+0)-u^{\prime}(-0)=2 U_{0} u_{0} .
\end{gathered}
$$

Таким образом, формулировка модели сводится к контактной краевой задаче для уравнений (5) и (6) с граничными условиями (7) и (8).

Следует отметить, что рассматриваемое уравнение вида (2) применимо не только для оптических систем. В частности, в работах $[29,30]$ уравнение такой формы использовалось для описания возникновения локализованных состояний в модели зонного антиферромагнетика с конгруэнтными сечениями поверхности Ферми. В данной интерпретации функция $u$ представляет собой обобщенный параметр порядка, описывающий распределение линейно поляризованных волн спиновой плотности. Также известно, что уравнение вида (2) формально эквивалентно уравнению Гросса-Питаевского, возникающему в теории конденсации Бозе-Эйнштейна [36-38]. В рамках этой теории выражение (4) интерпретируется как полное число частиц в системе и фактически играет роль условия нормировки.

\section{3. Локализация возмущений поля вдоль границ раздела линейной и нелинейной сред}

В данной работе будут рассматриваться только локализованные в пространстве решения сформулированной краевой задачи (5)-(8), которые удовлетворяют условию исчезновения на бесконечности $|u(x)| \rightarrow 0$ при $x \rightarrow \infty$. Такие решения описывают распределение светового поля в бегущей вдоль границы раздела сред волне и быстро затухающее при удалении от нее.
Несмотря на то, что подобные поверхностные волны на границе линейной и нелинейной сред рассматривались неоднократно [8-10], возникает необходимость подробного анализа влияния характеристик границ раздела сред на локализацию светового потока с позиций двух различных подходов, определяемых наборами параметров, физически реализуемых в конкретных экспериментальных ситуациях.

Рассмотрим далее два случая контакта линейной оптической среды с нелинейными средами с самофокусировкой и дефокусировкой.

\section{1. Нелинейные поверхностные волны вблизи границы раздела линейной и нелинейной самофокусирующей сред}

В случае контакта линейной среды с нелинейной самофокусирующей средой, характеризующейся положительной нелинейностью $\gamma>0$, при $\omega<\min \left\{\Omega_{L, N}\right\}$ существует решение УШ (5) и (6), удовлетворяющее граничным условиям (7) и (8):

$$
u(x)=\left\{\begin{array}{ll}
u_{0} e^{q_{L} x}, & x<0 \\
\frac{q_{N}}{\gamma^{1 / 2} \operatorname{ch}\left(q_{N}\left(x-x_{0}\right)\right)}, & x>0
\end{array},\right.
$$

где коэффициент пространственного затухания $q_{L}$ и волновое число $q_{N}$ определяются выражениями:

$$
q_{j}^{2}=2\left(\Omega_{j}-\omega\right)
$$

Здесь и далее значение индекса $j=L$ относится к характеристикам линейной среды при $x<0$, а $j=N-$ а нелинейной среды при $x>0$. Из (10) следует связь коэффициента пространственного затухания и волнового числа: $q_{N}^{2}=2 \Delta \Omega+q_{L}^{2}$, где $\Delta \Omega=\Omega_{N}-\Omega_{L}$. Амплитуда поля на границе раздела сред определяется после подстановки (9) в граничное условие (7):

$$
u_{o}=\frac{q_{N}}{\gamma^{1 / 2} \operatorname{ch}\left(q_{N} x_{0}\right)} .
$$

Подстановка (9) в нелинейное граничное условие (8) приводит к дисперсионному соотношению:

$$
q_{N} \text { th } q_{N} x_{0}-q_{L}=2 U_{0},
$$

из которого определяется связь константы распространения с характеристиками сред и границы их раздела.

Распределение поля (9) может иметь максимум на границе раздела сред или в нелинейной среде (справа от границы в рассматриваемой геометрии). Его положение определяется параметрами системы. Следовательно, получив возможность управления такими параметрами, можно регулировать расположение максимальной интенсивности поля.

В зависимости от постановки конкретных экспериментов можно выбирать один из возможных управляющих 
(свободных) параметров, в качестве которых могут выступать положение максимума возмущения поля в нелинейном полупространстве $x_{0}$ или значение амплитуды поля на границе раздела $u_{0}$.

Если сначала выбрать в качестве свободного параметра положение максимума возмущения поля в нелинейном полупространстве $x_{0}$, то из дисперсионного соотношения (12) можно определять зависимости коэффициента пространственного затухания поля $q_{L}$ и константы распространения $\omega$ от $x_{0}$, показателей линейных преломления сред и границы их раздела.

В частности, при $x_{0}=0$ из (12) находится коэффициент пространственного затухания поля $q_{L}=-2 U_{0}$, а затем из (10) находится константа распространения $\omega=\Omega_{L}-2 U_{0}^{2}$. Для данного случая локализация поля возможна только для притягивающей границы, так как коэффициент пространственного затухания должен быть положительным.

Для случая невзаимодействующей с волной границей и одинаковых параметрах сред при $U_{0}=0$ и $\Omega_{L}=\Omega_{N}$ получается $q_{L}=1 / x_{0}$ и $\omega=\Omega_{L}-1 / 2 x_{0}^{2}$. Отсюда следует, что характерное расстояние локализации поля вблизи границы совпадает с параметром $x_{0}$. Также получается, что положение максимума возмущения поля в нелинейном полупространстве в данном случае смещено вправо от границы раздела, так для положительности коэффициента пространственного затухания должно быть $x_{0}>0$.

В предельном случае $q_{N} x_{0} \ll 1$, который означает выполнения условия $\left|\Omega_{N}-\omega\right| \ll 1 / 2 x_{0}^{2}$, из (12) получается коэффициент пространственного затухания поля:

$$
q_{L}=\left\{1 \pm\left[1-8 x_{0}\left(\Delta \Omega x_{0}-U_{0}\right)\right]^{1 / 2}\right\} / 2 x_{0}^{2} .
$$

Знак в (13) выбирается так, чтобы значение коэффициента пространственного затухания было положительным. Для локализации такой нелинейной волны должно выполняться условие: $U_{0} \geq \Delta \Omega x_{0}-1 / x_{0}$, из которого следует, что локализация возможна вблизи притягивающей границы при условии $\Delta \Omega<1 / 8 x_{0}^{2}$, а вблизи отталкивающей границы - при условии $\Delta \Omega>1 / 8 x_{0}^{2}$. Критическое значение $U_{0}=\Delta \Omega x_{0}$ приводит к такому же коэффициенту пространственного затухания поля, который соответствует случаю невзаимодействующей с волной границей и одинаковых параметрах сред.

Если теперь выбрать в качестве свободного параметра амплитуду поля на границе раздела сред, то с помощью (11) можно исключить $x_{0}$ из (12) и получить точное решение дисперсионного уравнения в явном виде, соответствующее декременту затухания поля:

$$
q_{L}=\left(2 \Delta \Omega-4 U_{0}^{2}-\gamma u_{0}^{2}\right) / 2 U_{0} .
$$

Отсюда следует, что локализация поля возможна вблизи притягивающей границы при условии $u_{0}^{2}>2\left(\Delta \Omega-2 U_{0}^{2}\right) / \gamma$, а вблизи отталкивающей границы - при условии $u_{0}^{2}<2\left(\Delta \Omega-2 U_{0}^{2}\right) / \gamma$.

Можно отметить, что в случае одинаковых параметров сред (когда $\Delta \Omega=0$ ) из (14) получается
$q_{L}=-\left(4 U_{0}^{2}+\gamma u_{0}^{2}\right) / 2 U_{0}$, и видно, что локализация возможна только вблизи притягивающей границы. На основании этого можно сделать вывод о том, что одновременный учет различия линейных показателей преломления сред по разные стороны от границы раздела и взаимодействия волны с границей приводит к возможности существования нелинейных поверхностных волн как вблизи притягивающих, так и вблизи отталкивающих границ.

\section{2. Нелинейные поверхностные волны вблизи границы раздела линейной и нелинейной дефокусирующей сред}

Рассмотрим теперь случай отрицательного значения параметра нелинейности среды в полупространстве, когда $\gamma<0$ для всех $x>0$, что соответствует среде с дефокусировкой. В этом случае вблизи границы раздела линейной и нелинейной сред существует нелинейная поверхностная волна при $\omega<\min \left\{\Omega_{L, N}\right\}$, описываемая решением уравнений (5) и (6), удовлетворяющим граничным условиям (7) и (8):

$$
u(x)=\left\{\begin{array}{ll}
u_{0} e^{q_{L} x}, & x<0 \\
\frac{q_{N}}{g^{1 / 2} \operatorname{sh}\left(q_{N}\left(x-x_{0}\right)\right)}, & x>0
\end{array},\right.
$$

где $g=-\gamma>0$, коэффициент пространственного затухания и волновое число определяются выражением (10). Для ограниченности решения (15) должно выполняться дополнительное требование: $x_{0}<0$.

Амплитуда поля на границе раздела сред определяется после подстановки (15) в граничное условие (7):

$$
u_{0}=-\frac{q_{N}}{g^{1 / 2} \operatorname{sh}\left(q_{N} x_{0}\right)} .
$$

Подстановка (15) в нелинейное граничное условие (8) приводит к дисперсионному соотношению:

$$
q_{N} \operatorname{cth} q_{N} x_{0}+q_{L}=2 U_{0},
$$

из которого определяется связь константы распространения с характеристиками сред и границы их раздела.

Если сначала выбрать в качестве свободного параметра $x_{0}$, то из дисперсионного соотношения (17) можно определить зависимость коэффициента пространственного затухания поля $q_{L}$ и константы распространения $\omega$ от $x_{0}$, линейных показателей преломления сред и границы их раздела.

Из (17) следует, что нелинейные поверхностные волны в случае невзаимодействующей с волной границей и одинаковых параметрах сред $\left(U_{0}=0\right.$ и $\left.\Omega_{L}=\Omega_{N}\right)$ на границе линейной и дефокусирующей сред не существуют. Следовательно, для локализации светового потока на границе линейной и дефокусирующей сред необходимо наличие одного из указанных факторов.

В предельном случае $q_{N} x_{0} \ll 1$ из (17) получается коэффициент пространственного затухания поля 
$q_{L}=2 U_{0}-1 / x_{0}$, а затем из $(10)$ находится константа распространения $\omega=\Omega_{L}-\left(2 U_{0}-1 / x_{0}\right)^{2} / 2$. Для локализации такой нелинейной волны должно выполняться условие: $U_{0} \geq 1 / 2 x_{0}$, из которого следует, что локализация в этом случае возможна только вблизи притягивающей границы, поскольку было принято, что $x_{0}<0$.

В противоположном предельном случае $q_{N} x_{0} \gg 1$ для сред с одинаковыми линейными показателями преломления $\left(\Omega_{L}=\Omega_{N}\right)$ из (17) получается коэффициент пространственного затухания поля $q_{L}=U_{0}$, а затем из (10) находится константа распространения $\omega=\Omega_{L}-U_{0}^{2} / 2$. Для данного случая локализация поля возможна только для отталкивающей границы.

В этом же пределе для сред с различными линейными показателями преломления $\left(\Omega_{L} \neq \Omega_{N}\right)$ из (10) и (17) можно получить константу распространения в виде

$$
\omega=\left\{\Omega_{L} \Omega_{N}-\left(2 U_{0}^{2}-\Omega_{L}-\Omega_{N}\right)^{2} / 4\right\} / 2 U_{0}^{2} .
$$

Если теперь выбрать в качестве свободного параметра амплитуду поля на границе раздела сред, то с помощью (16) можно исключить $x_{0}$ из (17) и получить точное решение дисперсионного уравнения в явном виде, соответствующее коэффициенту пространственного затухания поля:

$$
q_{L}=\left(2 \Delta \Omega+4 U_{0}^{2}+g u_{0}^{2}\right) / 2 U_{0} .
$$

Отсюда следует, что локализация поля возможна вблизи притягивающей границы при условии $u_{0}^{2}=2\left(\Delta \Omega+2 U_{0}^{2}\right) / g$, а вблизи отталкивающей границы при условии $u_{0}^{2}<2\left(\Delta \Omega+2 U_{0}^{2} / g\right.$.

Можно отметить, что в случае одинаковых параметров сред (когда $\Delta \Omega=0$ ) из (19) получается $q_{l}=\left(4 U_{0}^{2}+g u_{0}^{2}\right) / 2 U_{0}$, и видно, что локализация возможна только вблизи отталкивающей границы, что отличает данное условие от условия локализации вблизи границы раздела линейной и нелинейной самофокусирующей сред. Также получается, что с ростом амплитуды поля на границе происходит уменьшение характерного расстояния локализации поля при удалении от границы.

\section{4. Поток энергии нелинейных поверхностных волн}

Вычисление потока энергии нелинейных поверхностных волн по формуле (4) приводит к выражению, которое можно представить в виде суммы двух частей:

$$
P=P_{L}+P_{N}
$$

где поток в линейном полупространстве при $x<0$ :

$$
P_{L}=u_{0}^{2} / 2 q_{L},
$$

и $P_{N}-$ поток в нелинейном полупространстве при $x>0$.

Форма (21) для потока в линейном полупространстве одинакова для нелинейных поверхностных волн обоих рассмотренных выше типов, поскольку в линейном полупространстве при $x<0$ зависимости поля от координаты согласно (9) и (15) одинаковы. Выражения для потоков в нелинейном полупространстве $P_{N}$ будут уже различаться.

Для анализа распределения между полупространствами потока энергии, уносимой нелинейной поверхностной волной, можно использовать относительный поток:

$$
\eta=P_{L} / P_{N}
$$

который можно будет представить в виде

$$
\eta=\eta_{0}+\eta_{b}
$$

где $\eta_{0}$ - относительный поток, соответствующий волне, не взаимодействующей с границей (не зависит от $U_{0}$ ), $\eta_{b}$ - относительный поток, соответствующий волне, которая взаимодействует с границей (зависит от $U_{0}$ ).

\section{1. Поток на границе раздела линейной и нелинейной самофокусирующей сред}

В случае контакта линейной среды с нелинейной самофокусирующей средой подстановка нелинейной поверхностной волны (9) в (4) приводит к выражению для потока в нелинейном полупространстве в виде

$$
P_{N}=q_{N}\left(1+\text { th } q_{N} x_{0}\right) / \gamma .
$$

Воспользовавшись дисперсионным соотношением (12), выражению (24) можно придать вид

$$
P_{N}=\left(q_{L}+q_{N}+2 U_{0}\right) / \gamma
$$

исключающий $x_{0}$.

Поток в линейной среде определяется выражением (21). Если воспользоваться еще (11) и исключить $u_{0}$, то выражение (21) примет вид

$$
P_{L}=\left\{\Delta \Omega-2 U_{0}\left(q_{L}+U_{0}\right)\right\} / \gamma q_{L} .
$$

Отсюда видно, что для случая не взаимодействующей с волной границей и одинаковых параметрах сред (когда $U_{0}=0$ и $\Delta \Omega=0$ ) получается, что поток в линейном полупространстве равен нулю, а поток в нелинейном полупространстве $P_{N}=2 / \gamma x_{0}$. Выражения (25) и (26) при $U_{0}=0$ и $\Delta \Omega \neq 0$ были приведены в [8].

Проанализируем зависимость потока от свободного параметра $x_{0}$. Сначала рассмотрим частный случай нелинейной поверхностной волны с максимумом распределения светового поля на границе раздела сред, когда $x_{0}=0$. В этом случае из (25), с учетом результатов анализа дисперсионного соотношения (12), получается

$$
\tilde{P}_{N}=P_{N}\left(x_{0}=0\right)=\left\{2\left(\Delta \Omega-2 U_{0}\right)\right\}^{1 / 2} / \gamma .
$$

Тогда суммарный поток можно представить в виде

$$
\tilde{P}=P\left(x_{0}=0\right)=\tilde{P}_{N}\left(1+\tilde{P}_{N} / \tilde{P}_{N}^{0}\right),
$$

где $\tilde{P}_{N}^{0}=-2 U_{0} / \gamma$. 
Из (28) в пределе сильного взаимодействия волны с границей при $|\Delta \Omega| \ll U_{0}^{2}$ можно получить оценку суммарного потока: $P=-\Delta \Omega / 2 \gamma U_{0}$. Получается, что в зависимости от соотношения между линейными показателями преломления сред поток может быть положительным как для притягивающей, так и для отталкивающей границы.

В случае одинаковых параметров сред (когда $\Delta \Omega=0$ ) при произвольном $x_{0}$ из $(25)$ и (26) получаются выражения

$$
\begin{gathered}
P_{L}=-2 U_{0}\left(1+U_{0} / q_{L}\right) / \gamma \\
P_{N}=2\left(q_{L}+U_{0}\right) / \gamma
\end{gathered}
$$

Если теперь дополнительно считать $x_{0}=0$, то из (29) и (30), с учетом результатов анализа дисперсионного соотношения (12), получается, что в линейном полупространстве поток нулевой: $P_{L}=0$, а в нелинейном: $P_{N}=\tilde{P}_{N}^{0}$, откуда следует, что поток положителен в среде с притягивающей границей.

Теперь проанализируем поток в предельном случае $q_{N} x_{0} \ll 1$. В этом случае из (21) и (24) получается выражение для суммарного потока в виде

$$
P=\tilde{P}+P_{x},
$$

где добавка к потоку, обусловленная наличием малого, но конечного значения управляющего параметра $x_{0}$ :

$$
P_{x}=\frac{2 x_{0}\left(\Delta \Omega+2 U_{0}^{2}\right)}{\gamma\left(1+4 x_{0} U_{0}\right)} .
$$

В случае слабого взаимодействия волны с границей раздела из (32) следует оценка добавки к потоку: $P_{x}=2 x_{0} \Delta \Omega / \gamma$. Если $x_{0}$ является положительным, то линейный показатель преломления в нелинейном полупространстве должен быть больше, чем в линейном, и наоборот, если $x_{0}$ является отрицательным, то линейный показатель преломления в нелинейном полупространстве должен быть меньше, чем в линейном.

В случае сильного взаимодействия волны с границей раздела из (32) следует оценка добавки к потоку: $P_{x}=U_{0} / \gamma$. Данная добавка к потоку положительна в случае отталкивающей границы.

Из всех рассмотренных случаев выше следует, что поток обратно пропорционален коэффициенту керровской нелинейности правого полупространства.

Из (25) и (26) можно получить относительный поток в форме (23) при слабом взаимодействии волны с границей раздела, где относительный поток, обусловленный взаимодействием волны с границей, будет иметь вид

$$
\eta_{0}=\left(q_{N} / q_{L}-1\right) / 2
$$

а относительный поток, соответствующий взаимодействующей с границей волне:

$$
\eta_{b}=-\frac{U_{0}}{q_{N}+q_{L}}\left(1+\frac{q_{N}}{q_{L}}\right) .
$$

При одинаковых линейных показателях преломления, как следует из (33), относительный поток, соответствующий волне, не взаимодействующей с границей, будет равен нулю. Следовательно, суммарный относительный поток (22) в рассматриваемом случае определяется только относительным потоком, обусловленным взаимодействием волны с границей, для которого из (34) получается оценка $\eta_{b}=-U_{0} / q_{L}$.

Проанализируем теперь зависимость потока на основе другого подхода, когда в качестве свободного параметра выбирается амплитуда светового поля на границе раздела сред $u_{0}$ (здесь фактически можно говорить об интенсивности светового потока вдоль границы, поскольку $\left.I=u_{0}^{2}\right)$.

Воспользовавшись зависимостью (14), выражению (26) для потока в линейном полупространстве можно придать вид

$$
P_{L}=\frac{U_{0} u_{0}^{2}}{2 \Delta \Omega-4 U_{0}^{2}-\gamma u_{0}^{2}},
$$

а выражению (25) для потока в нелинейном полупространстве:

$$
\begin{gathered}
P_{N}=P_{N 1}+P_{N 2}, \\
P_{N 1}=\left(8 \Delta \Omega U_{0}^{2}+\left(2 \Delta \Omega-4 U_{0}^{2}-\gamma u_{0}^{2}\right)^{2}\right)^{1 / 2} / 2 \gamma U_{0}, \\
P_{N 2}=\left(\left(2 \Delta \Omega-\gamma u_{0}^{2}\right) 4 U_{0}^{2}\right. \\
\left.+\left(2 \Delta \Omega-4 U_{0}^{2}-\gamma u_{0}^{2}\right)^{2}\right)^{1 / 2} / 2 \gamma U_{0} .
\end{gathered}
$$

Для малоамплитудных возмущений поля в пределе $u_{0}^{2} \ll\left(\Delta \Omega-2 U_{0}^{2} \mid / \gamma\right.$ из (35) получается выражение:

$$
P_{L}=\frac{U_{0} u_{0}^{2}}{2 \Delta \Omega-4 U_{0}^{2}},
$$

из которого для слабого взаимодействия волны с границей получается оценка потока в линейном полупространстве $P_{L}=U_{0} u_{0}^{2} / 2 \Delta \Omega$, а сильного: $P_{L}=-u_{0}^{2} / 4 U_{0}$.

Поток в нелинейном полупространстве для малоамплитудных возмущений поля в основном приближении не зависит от амплитуды поля на границе раздела и определяется выражением, вытекающим из (36):

$$
P_{N}=2\left(2 \Delta \Omega U_{0}^{2}+\left(\Delta \Omega-2 U_{0}^{2}\right)^{2}\right)^{1 / 2} / \gamma U_{0}
$$

Отсюда для слабого взаимодействия волны с границей получается оценка потока в нелинейном полупространстве: $P_{N}=2 \Delta \Omega / \gamma U_{0}$, а для сильного: $P_{N}=4 U_{0} / \gamma$.

Получается, что для малоамплитудных возмущений поля в случае слабого взаимодействия волны с границей поток в линейном полупространстве прямо пропорционален „мощности“ дефекта, а в нелинейном полупространстве - обратно пропорционален ей. В случае сильного взаимодействия волны с границей наоборот: поток в линейном полупространстве обратно пропорционален „мощности“ дефекта, а в нелинейном полупространстве - прямо пропорционален ей. 
Также можно получить оценки относительного потока (22) малоамплитудных возмущений поля для слабого взаимодействия волны с границей: $\eta=\gamma U_{0}^{2} u_{0}^{2} / 4 \Delta \Omega^{2}$, а для сильного: $\eta=-\gamma u_{0}^{2} / 16 U_{0}^{2}$. Получается, что для малоамплитудных возмущений поля в случае слабого взаимодействия волны с границей относительный поток всегда положительный, а для сильного - отрицательный.

В случае слабого взаимодействия волны с границей относительный поток пропорционален квадрату „мощности“ дефекта, а в случае сильного взаимодействия обратно пропорционален ему, а также не зависит от разности линейных показателей преломления сред. Оценки относительных потоков в двух предельных случаях показали также, что он прямо пропорционален коэффициенту керровской нелинейности нелинейной среды и квадрату амплитуды поля на границе раздела сред, т.е. интенсивности светового потока вдоль границы раздела сред.

\section{2. Поток на границе раздела линейной и нелинейной дефокусирующей сред}

В случае контакта линейной среды с нелинейной дефокусирующей средой подстановка нелинейной поверхностной волны (15) в (4) приводит к выражению для потока в нелинейном полупространстве в виде

$$
P_{N}=q_{N}\left(\operatorname{cth} q_{N} x_{0}-1\right) / g \text {. }
$$

Воспользовавшись дисперсионным соотношением (17), выражению (39) можно придать вид

$$
P_{N}=\left(2 U_{0}-q_{L}-q_{N}\right) / g,
$$

исключающий $x_{0}$.

Поток в линейной среде определяется выражением (21). Если воспользоваться (16) и исключить $u_{0}$, то выражение (21) примет вид

$$
P_{L}=\left\{2 U_{0}\left(q_{L}-U_{0}\right)-\Delta \Omega\right\} / g q_{L} .
$$

Проанализируем зависимость потока от свободного параметра $x_{0}$. Для этого (41) удобно представить в виде

$$
P_{L}=\frac{q_{N}^{2}}{2 g q_{L} \operatorname{sh}^{2}\left(q_{N} x_{0}\right)} .
$$

Сначала рассмотрим предельный случай $q_{N} x_{0} \ll 1$. Тогда из (42) получается оценка потока в линейной среде: $P_{L}=\left(2 g q_{L} x_{0}^{2}\right)^{-1}=\left\{2 g x_{0}\left(U_{0} x_{0}-1\right)\right\}^{-1}$, а из (39) 一 в нелинейной: $P_{N}=\left(g x_{0}\right)^{-1}$. Отсюда можно получить оценку относительного потока $\eta=\left(2 q_{L} x_{0}\right)^{-1}=\left\{2\left(U_{0} x_{0}-1\right)\right\}^{-1}$. Относительный поток в данном случае обратно пропорционален положению максимума возбуждения поля, а знак $\eta$ определяется знаками „мощности“ дефекта и $x_{0}$

В противоположном предельном случае при $q_{N} x_{0} \gg 1$ в основном приближении получается, что потоки в линейной и нелинейной средах пренебрежимо малы, но величина относительного потока при этом остается конечной: $\eta=q_{N} / q_{L}$, причем для случая одинаковых линейных показателях сред он равен единице.

Проанализируем теперь зависимость потока на основе второго подхода. Тогда, выбрав в качестве свободного параметра амплитуду светового поля на границе раздела сред $u_{0}$ и воспользовавшись зависимостью (19), выражению (40) для потока в линейном полупространстве можно придать вид

$$
P_{L}=\frac{U_{0} u_{0}^{2}}{2 \Delta \Omega+4 U_{0}^{2}+g u_{0}^{2}},
$$

а выражению (25) для потока в нелинейном полупространстве:

$$
\begin{gathered}
P_{N}=P_{N 1}+P_{N 2}, \\
P_{N 1}=-\left(8 \Delta \Omega U_{0}^{2}+\left(2 \Delta \Omega+4 U_{0}^{2}+g u_{0}^{2}\right)^{2}\right)^{1 / 2} / 2 g U_{0}, \\
P_{N 2}=-\left(\left(2 \Delta \Omega+g u_{0}^{2}\right) 4 U_{0}^{2}\right. \\
\left.+\left(2 \Delta \Omega+4 U_{0}^{2}+g u_{0}^{2}\right)^{2}\right)^{1 / 2} / 2 g U_{0} .
\end{gathered}
$$

Для малоамплитудных возмущений поля в пределе $u_{0}^{2} \ll\left|\Delta \Omega+2 U_{0}^{2}\right| / g$ из (43) получается выражение:

$$
P_{L}=\frac{U_{0} u_{0}^{2}}{2 \Delta \Omega+4 U_{0}^{2}},
$$

из которого для слабого взаимодействия волны с границей получается оценка потока в линейном полупространстве такая же, как и для поверхностной волны на границе с самофокусирующей средой, а сильного данная оценка потока будет противоположна по знаку соответствующей оценке, полученной в разд. 4.1.

Поток в нелинейном полупространстве для малоамплитудных волн в основном приближении не зависит от амплитуды поля на границе раздела и определяется выражением, вытекающим из (44):

$$
P_{N}=-2\left(2 \Delta \Omega U_{0}^{2}+\left(\Delta \Omega+2 U_{0}^{2}\right)^{2}\right)^{1 / 2} / g U_{0} .
$$

Отсюда для слабого взаимодействия волны с границей получается оценка потока в нелинейном полупространстве: $P_{N}=-2 \Delta \Omega / g U_{0}$, а для сильного: $P_{N}=-4 U_{0} / g$.

Далее на основе полученных выражений можно оценить относительный поток (22) малоамплитудных возмущений поля (15) для слабого взаимодействия волны с границей: $\eta=-g U_{0}^{2} u_{0}^{2} / 4 \Delta \Omega^{2}$ и для сильного: $\eta=-g u_{0}^{2} / 16 U_{0}^{2}$.

На качественном уровне выводы из полученных оценок потоков малоамплитудных волн на границе с дефокусирующей средой вытекают такие же, как и в разд. 4.1 для случая границы с самофокусирующей средой.

При использовании второго подхода к определению потоков видно, что все полученные оценки потоков, как для контакта линейной среды с самофокусирующей, так и с дефокусирующей средами, определяются квадратом амплитуды поля на границе, т. е. интенсивностью светового потока вдоль границы раздела сред. 


\section{5. Заключение}

В работе рассмотрено НУШ, которому подчиняется $y$-компонента напряженности электрического поля ТЕполяризованной электромагнитной волны, распространяющиеся вдоль границ раздела между линейными и нелинейными средами с положительным и отрицательным знаками параметра керровской нелинейности.

Для каждого случая получены дисперсионные соотношения, определяющие зависимости константы распространения от характеристик сред и границы их раздела. В частных предельных случаях получены декременты затухания нелинейных поверхностных волн в явном виде. Указаны различия условий локализации светового поля вблизи границ раздела линейной и самофокусирующей сред, а также линейной и дефокусирующей сред.

Основное внимание в работе уделено локализации светового потока вдоль границ линейной и нелинейной керровской сред при учете двух основных факторов, влияющих на существование поверхностных волн: разницы между значениями линейных показателей преломления сред и интенсивности взаимодействия волны с границей раздела сред. В зависимости от преобладания влияния одной из указанных величин возникают различные условия локализации нелинейных волн, а также оценки их параметров, таких как амплитуда поля на границе и декремент затухания волны при удалении от нее, определяющий характерное расстояние локализации светового потока.

Показано, что если в качестве управляющего процессом локализации параметра выбрать амплитуду поля на границе, появляется возможность регулировать характерное расстояние локализации поля вдоль границы.

Полученные дисперсионные соотношения применены для анализа влияния параметров сред и границы их раздела на поток, уносимый нелинейными поверхностными волнами.

В данной работе предложены два подхода к определению потока, зависящие от постановки конкретных экспериментов, когда можно выбирать один из возможных свободных параметров $x_{0}$ или $u_{0}$. В результате в явном виде получены зависимости потока $P=P\left(\mathbf{p}, x_{0}\right)$ и $P=P\left(\mathbf{p}, u_{0}\right)$, где $\mathbf{p}=\left\{\gamma, \Delta \Omega, U_{0}\right\}$ - вектор параметров, характеризующих среды и границу их раздела. К интерпретации данных зависимостей можно подойти с другой стороны, если рассматривать их как условия нормировки, считая поток фиксированным. Тогда можно считать поток управляющим параметром, через который выражаются положение максимума $x_{0}=x_{0}(\mathbf{p}, P)$ и амплитуда $u_{0}=u_{0}(\mathbf{p}, P)$. Получены оценки потоков в явном аналитическом виде для случаев слабого и сильного взаимодействий волны с границей раздела сред.

Полученные оценки потоков энергии, уносимых нелинейными поверхностными волнами, имеют значение для проектирования оптических волноводных систем с заданными характеристиками светопроводимости, а также оптических устройств управления на основе слоистых сред $[6,7]$.
Результаты анализа зависимостей потоков энергии поверхностных волн могут найти применение для совершенствования различных оптических переключателей в волноводах и оптических ограничителях мощности, способных пропускать световые импульсы только выше/ниже фиксированного значения потока энергии $[39,40]$. Комбинация поддающегося управлению порогового и ограничивающего действий может быть использована для определения оптимальных режимов передачи потоков энергии, которые несут нелинейные поверхностные волны.

\section{Список литературы}

[1] Kosevich A.M., Ivanov B.A., Kovalev A.S. // Phys. Rep. 1990. V. 194. P. 117. doi 10.1016/0370-1573(90)90130-T.

[2] Kivshar Yu.S.. Agrawal G.P. Optical Solitons: From Fibers to Photonic Crystals. Academic Press, San Diego, 2003. 540 р.; Кивщарь Ю.С, Агравал Г.П. Оптические солитоны. От волоконных световодов до фотонных кристаллов. М.: ФИЗМАТЛИТ, 2005. 648 с.

[3] Kartashov Y.V., Malomed B.A., Torner L. // Rev. Mod. Phys. 2011. V. 83. P. 247. doi 10.1103/RevModPhys.83.247.

[4] Carretero-González R., Cuevas-Maraver J., Frantzeskakis D., Karachalios N., Kevrekidis P., Palmero-Acebedo F. Localized Excitations in Nonlinear Complex Systems. Springer Science \& Business Media, 2013. 432 p.

[5] Kivshar Yu.S., Kosevich A.M., Chubykalo O.A. // Phys. Rev. A. 1990. V. 41. N 3. P. 1677. doi 10.1103/PhysRevA.41.1677.

[6] Паняев И.С., Санников Д.Г. // Компьютерная оптика. 2017. T. 41. № 2. C. 183-191. doi 10.18287/2412-6179-2017-41-2183-191.

[7] Strudley T., Bruck R., Mills B., Muskens O.L. Light: Science \& Applications 2014. N 3. P. e207. doi 10.1038/lsa.2014.88.

[8] Михалаке Д., Назмитдинов Р.Г., Федянин В.К. // Физика элементарных частиц и атомного ядра. 1989. Т. 20. № 1. C. 198.

[9] Ахмедиев Н.Н. // ЖЭТФ. 1982. Т. 83. С. 545. Akhmediev N.N. // Sov. Phys. JETP. 1982. V. 56(2). P. 299.

[10] Shadrivov I.V., Sukhorukov A.A., Kivshar Yu.S., Zharov A.A., Boardman A.D., Egan P. // Phys. Rev. E. 2004. V. 69. P. 016617-1. doi 10.1103/PhysRevE.69.016617.

[11] Bludov Y.V., Smirnova D.A., Kivshar Yu.S., Peres N.M.R., Vasilevsky M.I. // Phys. Rev. B. 2014. V. 89. P. 035406. doi 10.1103/PhysRevB.89.035406.

[12] Коровай О.В., Хаджи П.И. // ФТТ. 2010. Т. 52. С. 2277. Korovai O.V., Khadzhi P.I. // Phys. Solid State. 2010. V. 52. P. 2434. https://doi.org/10.1134/S106378341.

[13] Федоров Л.В., Ляхомская К.Д. // Письма в ЖТФ. 1997. T. 23. C. 36. Fedorov L.V., Ljahomskaja K.D. // Tech. Phys. Lett. 1997. V. 23. P. 915. doi 10.1134/1.1261931.

[14] Усиевич Б.А., Нурлигареев Д.Х., Сычугов В.А., Ивлева Л.И., Лыков П.А., Богодаев Н.В. // Квантовая электроника. 2010. Т. 40. № 5. С. 437. Usievich B.A., Nurligareev D.Kh., Sychugov V.A., Ivleva L.I., Lykov P.A., Bogodaev N.V. // Quantum Electronics. 2010. V. 40. P. 437. doi 10.1070/QE2010v040n05ABEH014223.

[15] Sukhorukov A.A., Kivshar Yu.S. // Phys. Rev. Lett. 2001. V. 87. P. 083901. doi 10.1103/PhysRevLett.87.083901. 
[16] Савотченко C.E. // Конденсированные среды и межфазные границы. 2017. Т. 19. № 4. С. 567. doi 10.17308/kcmf.2017.19/238.

[17] Савотченко C.E. // Вестник Воронежского государственного университета. Серия: Физика. Математика. 2018. № 1. C. 44.

[18] Савотченко C.E. // ЖЭТФ. 2018. Т. 153. № 2. С. 339. Savotchenko S.E. // JETP. 2018. V. 126. N 2. P. 284. doi: $10.1134 / \mathrm{S} 1063776118020061$.

[19] Савотченко C.E. // Конденсированные среды и межфазные границы. 2017. Т. 19. № 2. С. 291. doi $10.17308 / \mathrm{kcmf} .2017 .19 / 205$.

[20] Савотченко C.E. // ЖТФ. 2017. Т. 62. № 12. С. 1776-1781. Savotchenko S.E. // Tech. Phys. 2017. V. 62. N 2. P. 1772. doi 10.1134/S1063784217120210.

[21] Savotchenko S.E. // Modern Physics Letters B. 2018. V. 32. N 19. P. 1850222. doi 10.1142/S0217984918502226.

[22] Савотченко C.E. // Известия вузов. Физика. 2004. Т. 47. № 5. C. 79-84. Savotchenko S.E. // Russian Physics Journal. 2004. V. 47. N 5. P. 556. doi 10.1023/B:RUPJ.0000046330.92744.73.

[23] Богдан М.М., Герасимчук И.В., Ковалев А.С. // ФНТ. 1997. T. 23. № 2. C. 197. Bogdan M.M., Gerasimchuk I.V., Kovalev A.S. // Low Temp. Phys. 1997. V. 23. P. 197. doi 10.1063/1.593346.

[24] Gerasimchuk I.V., Gorbach P.K., Dovhopolyi P.P. // Ukr. J. Phys. 2012. V. 57. N 6. P. 678.

[25] Герасимчук И.В. // ЖЭТФ. 2015. Т. 121. № 4. С. 596. Gerasimchuk I.V. // JETP. 2015. V. 121. N 4. P. 596. doi 10.1134/S1063776115100076.

[26] Савотченко С.Е. // Письма в ЖЭТФ. 2018. Т. 107. № 8. C. 481. Savotchenko S.E. // JETP Lett. 2018. V. 107. N 8. P. 455. doi 10.7868/S0370274X18080027.

[27] Savotchenko S.E. // Mod. Phys. Lett. B. 2018. V. 32. N 10. P. 1850120. doi 10.1142/S0217984918501208.

[28] Савотченко C.E. // Конденсированные среды и межфазные границы. 2018. Т. 20. № 2. С. 255. doi 10.17308/kcmf.2018.20/517.

[29] Савотченко С.Е. // Письма в ЖЭТФ. 2018. Т. 108. № 3. C. 175. Savotchenko S.E. // JETP Lett. 2018. V. 108. N 3. P. 175. doi 10.1134/S0021364018150110.

[30] Savotchenko S.E. // Solid State Commun. 2018. V. 283. N 11. P. 1. doi 10.1016/j.ssc.2018.08.002.

[31] Косевич А.М., Ковалев А.С. Введение в нелинейную физическую механику. Киев: Наукова думка, 1989. 304 с.

[32] Горенцвейг В.И., Кившарь Ю.С., Косевич А.М., Сыркин E.C. // ФНТ. 1990. Т. 16. № 11. С. 1472.

[33] Косевич А.М., Иванов Б.А., Ковалев А.С. Нелинейные волны намагниченности. Динамические и топологические солитоны. Киев: Наукова думка, 1983. 189 с.

[34] Давыдов А.С. Солитоны в молекулярных системах. Киев: Наукова думка, 1984. 288 с.

[35] Kivshar Yu.S., Kosevich A.M., Chubykalo O.A. // Phys. Lett. A. 1987. V. 125. P. 35. doi 10.1016/0375-9601(87)90514-7.

[36] Sakaguchi H., Malomed B.A. // New J. Phys. 2016. V. 18. P. 025020 . doi $10.1088 / 1367-2630 / 18 / 2 / 025020$.

[37] Чаплик А.В. // Письма в ЖЭТФ. 2017. Т. 105. С. 565. Chaplik A.V. // JETP Lett. 2017. V. 105. P. 601. doi 10.1134/S0021364017090089.
[38] Высотина Н.В., Розанов Н.Н., Шацев А.Н. // Опт. и спектр. 2018. T. 124. № 1. C. 82. Vysotina N.V., Rosanov N.N., Shatsev A.N. Optics and Spectroscopy. 2018. V. 124. P. 79. doi 10.1134/S0030400X18010228.

[39] Zhang D., Li Z., Hu W., Cheng B. // Appl. Phys. Lett. 1995. V. 67. P. 2431. doi 10.1063/1.114597.

[40] Naim Ben Ali // Chinese J. Phys. 2017. V. 55. P. 2384. doi 10.1016/j.cjph.2017.10.008. 